\title{
Training Effects Outweigh Effects of Single-Session Conventional rTMS and Theta Burst Stimulation in PD Patients
}

\author{
Holger Rothkegel, Martin Sommer, MD, Thomas Rammsayer, PhD, \\ Claudia Trenkwalder, MD, and Walter Paulus, MD
}

\begin{abstract}
Background. Focal single-session repetitive transcranial magnetic stimulation (rTMS) of the primary motor cortex has been claimed to be capable of improving motor function in Parkinson's disease. Objective. The authors sought to determine which type of rTMS protocol holds the highest potential for future therapeutic application. Methods. Twenty-two patients with Parkinson's disease received 5 different rTMS protocols on 5 consecutive days in a pseudorandomized and counterbalanced order either in the defined OFF condition or with their usual medication. The protocols tested in the present study included 2 conventional rTMS protocols $(0.5 \mathrm{and} 10 \mathrm{~Hz})$ as well as the recently introduced theta burst stimulation (cTBS, iTBS) and a sham condition. Cortical excitability, motor performance (pointing movement, pronation-supination, Purdue Pegboard Test, walking), and mood were assessed before and after each session. Results. The authors observed motor training from days 1 to 4, particularly in the group on dopaminergic medication. None of the rTMS paradigms excelled placebo stimulation. The only exception was the Purdue Pegboard Test, in which all active stimulation paradigms yielded slightly stronger effects than sham stimulation. Conclusions. Within a single session, no clinically relevant difference in the rTMS protocols could be detected. Training effects outweigh and may have masked rTMS effects, particularly in the group on dopaminergic mediation.
\end{abstract}

Keywords: Parkinson's disease; Single-session repetitive transcranial magnetic stimulation (rTMS); Theta burst stimulation (TBS).

$\mathrm{D}$ opaminergic drugs are a highly effective treatment in the initial stage of Parkinson's disease (PD). However, gait disorder, on-freezing, and postural instability do not respond well to dopaminergic treatment. With disease progression, the dopaminergic drug effects shrink, with response fluctuations and akinesia on one side and sometimes painful and disabling dyskinesias on the other side. Therefore, a number of alternative, nonpharmacological procedures have been suggested.

Repetitive transcranial magnetic stimulation (rTMS) is a noninvasive technique that is capable of inducing alterations of neuronal network excitability in the area directly targeted by the stimulation coil as well as in connected areas outlasting the time of stimulation. ${ }^{1,2}$ Even with focal stimulation over the motor cortex, remote areas such as the basal ganglia can be affected. ${ }^{3}$ Therefore, rTMS has been assumed as a tool possibly restoring pathologically altered excitability of cerebral motor areas in movement disorders. A multitude of different stimulation paradigms varying in frequency, intensity, configuration, or location of rTMS has already been tested in PD patients. However, the results so far have been modest and inconsistent $^{4-7}$ (for review, see Fregni et al, ${ }^{8}$ Helmich et al, ${ }^{9}$ and Sommer and Paulus ${ }^{10}$ ). None of the protocols so far has made its way into standard therapy.

Recently, theta burst stimulation (TBS) has been adapted as a new TMS protocol. ${ }^{11}$ Theta burst stimulation seems to have a higher potential of inducing stronger and more reliable aftereffects than conventional rTMS, thus possibly making it a better option for treatment studies in movement disorders. ${ }^{12}$ Because it is not possible to predict the effects of neuroplasticity-inducing protocols on cortical areas with pathologically altered excitability from studies on young healthy subjects alone, ${ }^{4,13}$ we have studied TBS effects in PD patients.

The aims of this study were to assess whether (1) a single session of TBS was able to improve motor performance in PD immediately after stimulation, (2) TBS was more effective than conventional rTMS, (3) unilateral stimulation of the hand motor area had differential effects on contralateral hand muscles compared with other muscle groups, (4) dopaminergic medication was necessary to achieve these effects, and (5) effects on motor performance were associated with changes in cortical excitability.

From the Department of Clinical Neurophysiology, University of Göttingen, Göttingen, Germany (HR, MS, WP); Paracelsus Elena-Klinik, Kassel, Germany (CT); Georg-Elias-Müller Institute for Psychology, Göttingen, Germany (TR); and University of Bern, Institute for Psychology, Bern, Switzerland (TR). Address correspondence to W. Paulus, MD, Department of Clinical Neurophysiology, University of Göttingen, Robert-Koch-Straße 40, 37075 Göttingen, Germany. E-mail: wpaulus@med.uni-goettingen.de. 
Table 1

Clinical Features and Antiparkinsonian Medication

\begin{tabular}{|c|c|c|c|c|c|c|c|c|c|c|}
\hline & Gender & Age, y & Duration & Symptoms & UPDRS III & L-Dopa, mg & $\begin{array}{l}\text { Intakes } \\
\text { Per Day }\end{array}$ & Agonists & LED, mg & Other \\
\hline \multicolumn{11}{|l|}{ OFF-group } \\
\hline 2 & $\mathrm{~F}$ & 69 & 38 & ART & 28 & 350 & 9 & $\mathrm{Cab} 4 \mathrm{mg}$, Pram $2.1 \mathrm{mg}$ & 800 & Ent, Aman \\
\hline 3 & $\mathrm{~F}$ & 64 & 8 & ART & 14 & 350 & 6 & Cab 6 mg & 650 & \\
\hline 4 & M & 52 & 3 & ART & 18 & 300 & 4 & Rop 20 mg & 800 & \\
\hline 5 & M & 65 & 6 & ART & 52 & 825 & 8 & Pram $2.45 \mathrm{mg}$ & 1115 & Tol, Aman \\
\hline 8 & M & 65 & 3 & ART & 23 & 400 & 4 & Cab $6 \mathrm{mg}$ & 700 & \\
\hline 9 & M & 67 & 7 & ART & 17 & 500 & 5 & $\mathrm{Cab} 4.5 \mathrm{mg}$ & 725 & \\
\hline 10 & $\mathrm{~F}$ & 66 & 7 & ART & 20 & 675 & 8 & Pram $2.45 \mathrm{mg}$ & 965 & Ent, Aman, Ras \\
\hline 11 & $\mathrm{~F}$ & 73 & 4 & $\mathrm{AR}$ & 11 & 800 & 9 & - & 800 & Ent \\
\hline Mean & F/M: & 67.0 & 10.4 & & 27.0 & 572.7 & 6.7 & & 833.2 & \\
\hline $\mathrm{SD}$ & $5 / 6$ & 6.4 & 11.0 & & 13.9 & 214.3 & 2.0 & & 175.7 & \\
\hline 15 & M & 48 & 1 & AR & 15 & 100 & 2 & Pram $1.4 \mathrm{mg}$ & 265 & Ras, Aman \\
\hline 16 & M & 65 & 13 & $\mathrm{AR}$ & 38 & 650 & 8 & Rop 6 mg & 800 & Ent \\
\hline 17 & M & 59 & 8 & $\mathrm{AR}$ & 18 & 1100 & 9 & $\begin{array}{l}\text { Cab } 4 \text { mg, Pram } 1.62 \mathrm{mg} \text {, } \\
\text { Apo } 4 \text { mg ( } 2 \text { days) }\end{array}$ & $\begin{array}{c}1490 \\
(1590)\end{array}$ & Ent, Ras, Aman \\
\hline 18 & $\mathrm{~F}$ & 63 & 17 & ART & 46 & 1000 & 9 & Pram $2.1 \mathrm{mg}$ & 1350 & Ent, Aman \\
\hline 19 & M & 34 & 6 & ART & 21 & 0 & 0 & Cab 6 mg, Pram $2.1 \mathrm{mg}$ & 550 & Ras, Aman \\
\hline 20 & $\mathrm{~F}$ & 65 & 7 & $\mathrm{AR}$ & 21 & 350 & 8 & Cab $3 \mathrm{mg}$ & 500 & \\
\hline 21 & M & 56 & 5 & ART & 17 & 575 & 8 & Pram $2.8 \mathrm{mg}$ & 905 & Ras \\
\hline 22 & M & 65 & 8 & ART & 32 & 675 & 6 & Pram $1.75 \mathrm{mg}$ & 880 & Bud \\
\hline Mean & F/M: & 61.1 & 8.7 & & 26.7 & 572.7 & 6.6 & & 865.5 & \\
\hline SD & $5 / 6$ & 11.9 & 4.3 & & 10.4 & 345.8 & 3.0 & & 398.2 & \\
\hline Overall Mean & F/M: & 64.0 & 9.5 & & 26.9 & 572.7 & 6.7 & & 849.3 & \\
\hline
\end{tabular}

Abbreviations: M, male; F, female; duration, duration since disease onset in years. Symptoms, dominant symptoms of Parkinson's Disease; AR, akinetic-rigid; ART, akinetic-rigid-tremor; UPDRS III, Unified Parkinson's Disease Rating Scale part III motor score. Dopamine agonists and daily dose: Pram, pramipexol; Cab, cabergoline; Rop, ropinirol; Apo, apomorphine; LED, L-Dopa equivalent dose. Other antiparkinsonian drugs: Ent, entacapone; Tol, tolcapone; Ras, rasagiline; Aman, amantadine; Bud, budipine.

\section{Material and Methods}

\section{Subjects}

Twenty-two patients were studied while inpatients at the Paracelsus-Elena-Klinik, Kassel. All patients fulfilled the UK Parkinson's Disease Brain Bank Criteria for PD and were in Hoehn \& Yahr stages II to IV. No antiparkinsonian medication was newly introduced or stopped during the trial week, and slight changes of the dosage were allowed according to the patients' needs. Patients with severe motor fluctuations, dementia, or any contraindication against TMS (metal or electronic implants, cerebral ischemia, epilepsy, instable psychiatric or internal diseases, pregnancy, drug or alcohol abuse) were excluded. Depression, a frequent comorbidity in this population, was evaluated using the Beck Depression Inventory ${ }^{14}$ with 5 patients in the OFF group and 4 patients in the $\mathrm{ON}$ group, yielding scores higher than 11 . None of the subjects had ongoing psychosis or hallucinations at the time of the study.
All patients gave written informed consent to the study protocol, which had been approved by the ethics committees of the University of Göttingen and the Landesärztekammer Hessen and was in accordance with the Declaration of Helsinki.

\section{General Study Design}

To study the influence of dopaminergic medication, we decided to randomize patients to receive rTMS either in the defined OFF condition in the morning (ie, after a 12-hour overnight withdrawal of antiparkinsonian medication ${ }^{15}$; OFFgroup) or as add-on intervention while continuing to take their normal medication (ON-group). The 2 groups did not differ significantly in gender, age, duration, and dominant PD symptom (tremor or hypokinesia), dose of L-dopa, or L-dopa equivalent dose (see Table 1), as shown by 2-tailed unpaired $t$ tests or chi-square tests where appropriate (SPSS 12.0 for Windows, SPSS Inc, Chicago, Illinois). A $P$ value of $<.05$ was considered significant for all statistical tests. L-dopa equivalent dose was calculated according to the guidelines of the 
German Parkinson-Network (www.kompetenznetz-parkinson. de/Parkinson/leittherapie.html, retrieved July 11, 2006: L-dopa equivalent dose $=$ L-dopa + apomorphine $/ 4 * 100+$ cabergoline $/ 2 * 100+$ pramipexole $/ 0.85 * 100+$ ropinirole $/ 4 *$ 100 [daily doses]).

All patients had one training session encompassing all clinical tests before entering the study to prevent strong learning effects in the motor tasks and to familiarize them with the procedures of TMS. Five different rTMS protocols, including sham stimulation, were tested on 5 consecutive days in a pseudorandomized order at the same time of day for each subject. Patients were told that different rTMS protocols were about to be studied in the search of the optimal clinical effect, but no details were given about the different types of stimulation or about the presence of a sham condition.

\section{Intervention}

rTMS was generated by a Medtronic MagPro X100 + MagOption stimulator in the biphasic mode with reversed current direction (initially posterior-anterior current flow in the brain, as originally described for $\mathrm{TBS}^{11}$ ). A slightly bent figure-of-8 coil (Medtronic MC-B70) was held perpendicular to the head over the optimal representation of the target muscle (see assessment of corticospinal excitability) with the handle pointing posteriorly and 45 degrees laterally.

We chose $0.5 \mathrm{~Hz}$ (continuously, 600 pulses at an intensity of $80 \%$ resting motor threshold [RMT]) and $10 \mathrm{~Hz}$ (20 trains of 100 pulses, 50-second intertrain interval, $80 \%$ RMT) as conventional rTMS protocols, as published by Lefaucheur et al. ${ }^{5}$ For TBS, we used the continuous (cTBS, 600 pulses, $80 \%$ active motor threshold [AMT]) and intermittent (iTBS, 20 trains of 30 pulses, 8 -second intertrain interval) pattern, as described by Huang et al. ${ }^{11}$ In brief, TBS stimulation consists of triplets of pulses at a high frequency $(50 \mathrm{~Hz})$ repeated with a lower frequency in the theta range $(5 \mathrm{~Hz})$. For sham intervention, we used the iTBS protocol with the coil tilted at 90 degrees so that only the edge of the coil touched the head. ${ }^{16}$

\section{Assessment}

Immediately before the first and after the last session, patients were assessed using the motor section of the Unified Parkinson's Disease Rating Scale (UPDRS), ${ }^{17}$ and they completed the Beck Depression Inventory (BDI) ${ }^{14}$ to test for changes during the week of experiments.

Corticospinal excitability, several motor tasks, and a behavioral self-rating scale were assessed in the following order starting 5 minutes after intervention and, for practical reasons, in reversed order for baseline measurements:

1. Corticospinal excitability (RMT, AMT, motor evoked potential [MEP], background electromyographic [EMG] activity)

2. Rapidly alternating arm movements (pointing task, pronationsupination)

3. Purdue Pegboard Task (PPT)
4. Mood self-rating scale

5. Gait (time, number of steps)

(1) Corticospinal excitability. Surface EMG was recorded with $\mathrm{Ag} / \mathrm{AgCl}$ cup electrodes in a belly-tendon montage from the abductor digiti minimi (ADM) of the more affected hand. Signals were amplified with a Toennies Electromyograph II (Toennies, Würzburg, Germany) using a bandpass filter of 1.6 to $1000 \mathrm{~Hz}$, sampled with a CED Micro $1401 \mathrm{mk}$ II (Cambridge Electronic Design, Cambridge, England) at a rate of $5 \mathrm{kHz}$ and stored on a lab computer for offline analysis. Single-pulse TMS was applied using the Medtronic stimulator with the same settings as for the interventions (biphasic pulses, reversed current direction). The coil was moved over the assumed location of the primary motor cortex contralateral to the more affected side of the body. The point where maximum responses in the ADM were observed was defined as the optimal cortical representation of this muscle and was used for single-pulse and repetitive stimulation.

Resting motor threshold was determined as the minimum intensity at which at least 5 out of 10 consecutive TMS pulses induced MEPs of $>50 \mu \mathrm{V}$ in amplitude with the subject at rest. Active motor threshold was measured under tonic contraction of the target muscle of about $20 \%$ of maximum EMG activity, as monitored by visual feedback. The minimum intensity at which at least 5 out of 10 TMS pulses induced MEPs of $>200 \mu \mathrm{V}$ in amplitude was considered AMT. ${ }^{18}$

For assessment of MEP amplitudes, 20 single pulses were applied every $4 \pm 0.4$ seconds. The intensity of the magnetic pulses was adjusted to induce MEPs of about $1 \mathrm{mV}$ at baseline and kept constant for the measurement after intervention. Peak-to-peak amplitudes were measured offline.

Mean baseline EMG activity was measured in the $80 \mathrm{~ms}$ preceding the TMS stimulus. Pearson's correlation coefficients were calculated to investigate the relations between baseline EMG activity, motor thresholds, and overall rigidity, as well as rigidity of the more affected arm (ie, contralateral to the stimulated hemisphere), as assessed by the respective UPDRS score on the first day of experiments.

(2) Rapidly alternating arm movements. Two types of rapidly alternating arm movements were recorded with an ultrasound-based 3D motion analysis system (zebris CMS-HS using customized WinArm Software, zebris Medical $\mathrm{GmbH}$, Isny im Allgäu, Germany).

First, an arm-hand pointing movement between 2 targets $30 \mathrm{~cm}$ apart was performed according to the Core Assessment Program for Surgical Interventional Therapies in Parkinson's Disease $\left(\right.$ CAPSIT-PD $\left.^{15}\right)$ and recorded at a sampling rate of $100 \mathrm{~Hz}$.

Second, a forearm pronation/supination movement was assessed using the predefined item of the WinArm Software (sampling rate $80 \mathrm{~Hz}$ ).

We recorded 2 trials for either hand at each time point. Motion trajectories were analyzed offline for average frequency and amplitude of 8 full-movement cycles, leaving out the first 2 
cycles (starting phase). As the frequency of rapid, alternating movements depends on the amplitude, we chose movement speed (ie, product of mean frequency and mean amplitude) as a more reliable parameter. Results for each sample were normalized to the individual baseline of each experiment.

(3) Purdue Pegboard Task. As a test for fine motor skills and complex upper limb movements, we tested performance with the PPT (Lafayette Instrument Co. Europe, Loughborough, UK) for both hands separately and bimanually. Patients were instructed to pick up pins from a cup and place them in holes in the board starting with the top hole as fast as possible for 30 seconds. Two trials for either hand and for the bimanual task were performed, and the number of pins placed correctly was counted.

(4) Mood self-rating scale. Before and after each session, subjects completed a self-rating questionnaire containing the following items as 7-point scales ranging from -3 to +3 , adapted and translated from Strafella et $\mathrm{al}^{3}$ : comfort, anxiety, fatigue, mood, irritation, attention, and pain. Positive values represent positive feelings, whereas negative values represent negative feelings. After stimulation, subjects were asked whether they felt the respective protocol to be effective for them and whether they felt better, worse, or the same as before.

(5) Gait performance. Patients were asked to perform the walking test of the CAPSIT-PD. ${ }^{15}$ We measured the duration and counted the number of steps for walking 7 meters forth and back, including turning ( 2 trials at each time point). All values were normalized to the individual baseline of each experiment.

\section{Statistical Analysis}

For UPDRS and BDI scores, repeated-measures analyses of variance (ANOVAs) were calculated with the 2 time points (before/after the week of stimulation) as the within-subjects factor and group (ON/OFF) as the between-subjects factor. We also tested for baseline differences between the 2 groups using 2-tailed unpaired $t$ tests.

To test for different effects of the 5 intervention protocols $(0.5 \mathrm{~Hz}, 10 \mathrm{~Hz}$, iTBS, cTBS, sham), we performed repeatedmeasures ANOVAs (Statview 5.0, SAS Institute Inc, Cary, North Carolina) for all tests separately with intervention protocol and group (ON/OFF) as between-subjects factors. Within-subjects factors were time (pre/post), trial (for all motor tasks), and hand (only for arm movement tasks). For motor thresholds, the level of activation was used as an additional within-subjects factor. The intraindividual variance of MEP amplitudes was too high to allow for meaningful statistical analysis, which we therefore omitted. Results of the Purdue Pegboard Task were entered into separate repeated-measures ANOVAs for the unimanual and the bimanual tasks.

The 7-point self-rating scales were tested for changes after intervention or over the week of experiments by ANOVAs as described above for the motor tasks. The 2 items, efficacy and general change, were tested using chi-square tests for crosstable relationships.

To further address the question of training effects over the week of experiments, we performed ANOVAs on the nonnormalized values of each test with group as the between-subjects factor and day (+ trial and hand, where applicable) as withinsubjects factors.

\section{Results}

The behavioral measures showed a remarkable training effect, with gradual performance improvement from day 1 to day 4 . This training effect was particularly pronounced in the group of patients "on" dopaminergic medication.

Rapidly alternating movements. In both groups, performance in the arm-hand pointing task improved in either hand after intervention and from trial 1 to trial 2 (ANOVA, effect of time, $F(1,100)=11.050, P=.0012$; effect of trial, $F(1,100)=35.087$, $P<.0001)$. This improvement was particularly pronounced for the more affected side in the ON-group (Interaction Side $\times$ Group, $F(1,100)=6.912, P=.0099$; Interaction Side $\times$ Time $\times$ Group, $F(1,100)=6.862, P=.0102$; Interaction Side $\times$ Trial $\times$ Group, $F(1,100)=7.225, P=.0084$; see Figure 1a).

Analysis of baseline raw values revealed increasing performance during the first 4 days of the study week for both groups (ANOVA, effect of day, $F(4,80)=16.544, P<.0001$ ), which was more pronounced in the ON-group (Interaction Day $\times$ Group, $F(4,80)=2.800, P=.0313$; see Figure $1 b$ ). Performance in trial 2 was generally better than in trial 1 (effect of trial, $F(1,20)=11.160, P=.0033$ ), with the steepest increase for the more affected hand in the ON-group (Interaction Side $\times$ Trial $\times$ Group, $F(1,20)=4.374, P=.0495)$.

For the forearm pronation/supination task in the ON-group but not in the OFF-group, we found an improved performance after intervention, as measured by the product of frequency and amplitude normalized to baseline (ANOVA, effect of group, $F(1,100)=4.281, P=.0411$; effect of time, $F(1,100)=$ 4.784, $P=.0311$; Interaction Time $\times$ Group, $F(1,100)=3.920$, $P=.0505)$. Performance in the second trial compared with the first trial was reduced in the ON-group (effect of trial, $F(1$, $100)=11.873, P=.0008$; Interaction Trial $\times$ Group, $F(1,100)=$ $5.596, P=.0199)$ for both the stimulated and nonstimulated sides before and after intervention (no interaction of trial with side or time), whereas performance in the OFF-group was rather constant in both trials (see Figure 2a).

Over the week of experiments, the ON-group's baseline performance improved during the first days, whereas there was a slight decrease in performance for the OFF-group (ANOVA for baseline raw values, Interaction Day $\times$ Group, $F(4,80)=$ $5.113, P=.0010$; see Figure $2 b)$. Trial 2 was generally worse than trial 1 (effect of trial, $F(1,20)=7.279, P=.0138$ ), only for the first day, this pattern was reversed (Interaction Day $X$ Trial, $F(4,80)=2.649, P=.0392)$. 
Figure 1

Arm-Hand Pointing Movement

a

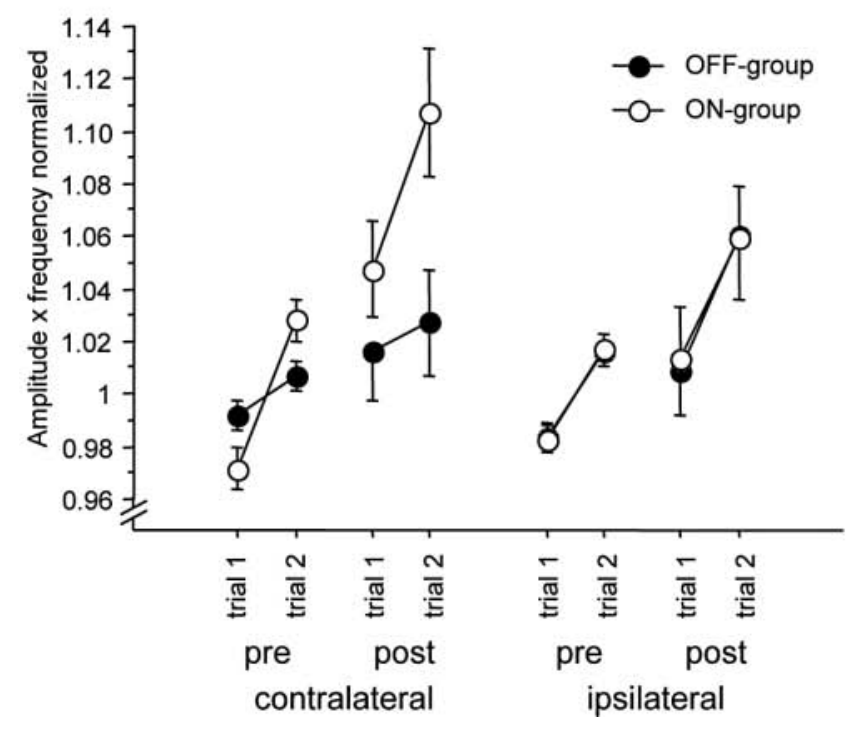

b

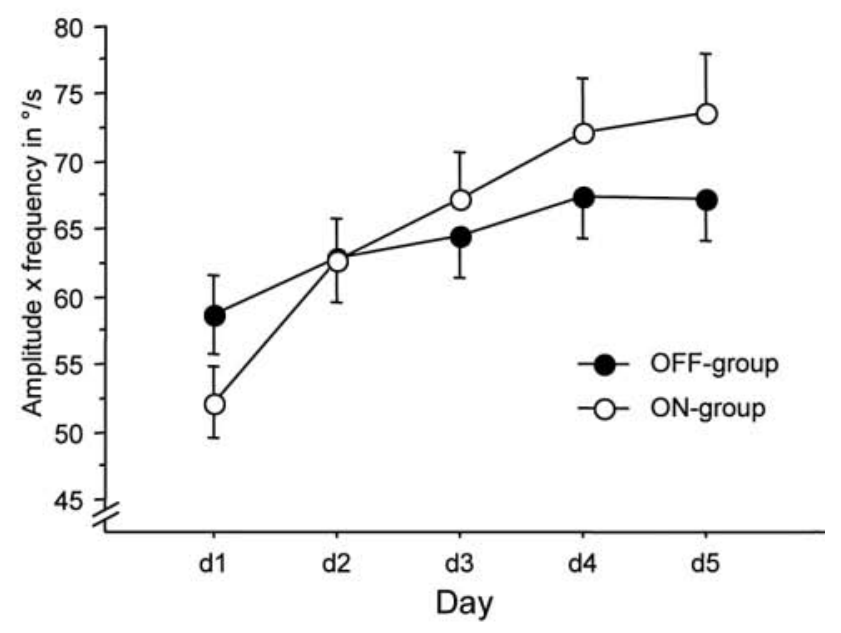

Note: Higher values indicate better performance. Results of the different types of intervention are pooled because analysis of variance (ANOVA) did not show any main effect or interaction for this factor. (a) Amplitude $\times$ Frequency normalized to individual mean baseline values (pretrials 1 and 2); (b) Amplitude $\times$ Frequency, mean baseline values over the days of the experiment, both sides pooled; error bars indicate \pm 1 SEM.

Figure 2

\section{Forearm Pronation-Supination Movement}
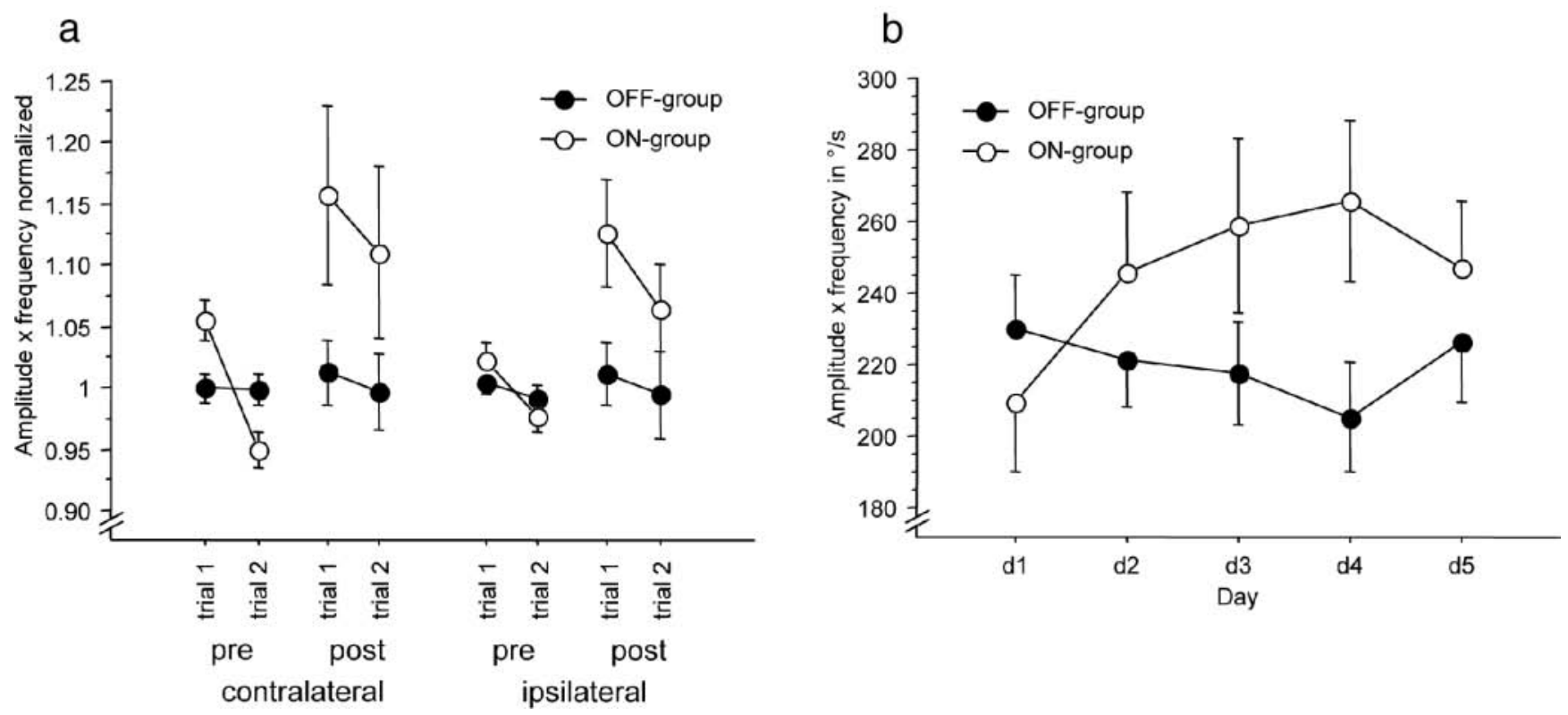

Note: Higher values indicate better performance. Results of the different types of intervention are pooled because analysis of variance (ANOVA) did not show any main effect or interaction for this factor. (a) Amplitude $\times$ Frequency normalized to individual mean baseline values (pretrials 1 and 2); (b) Amplitude $\times$ Frequency, mean baseline values over the days of the experiment, both sides pooled; error bars indicate \pm 1 SEM. 
$P P T$. Performance in the unimanual tasks improved after intervention (ANOVA, effect of time, $F(1,95)=5.161 P=.0254$; see rTMS-related effects). Performance also improved from trial 1 to trial 2 (effect of trial, $F(1,95)=49.414, P<.0001$ ) in the ON-group, especially at baseline (Interaction Time $\times$ Trial $\times$ Group, $F(1,95)=5.121, P=.0259$; Time $\times$ Trial, $F(1,95)=$ 4.067, $P=.0466)$.

For the bimanual task, performance improved after intervention and from trial 1 to trial 2 (ANOVA, effect of time, $F(1,95)=7.355, P=.0079$; effect of trial, $F(1,95)=12.047$, $P=.0008)$.

Baseline values in the unimanual task improved during the week of experiments (ANOVA, effect of day, $F(4,76)=9.363$, $P<.0001)$, from $9.24 \pm 2.00$ (mean \pm SD) pins to $10.41 \pm 1.86$ pins on the more affected side and from $10.29 \pm 1.88$ to 11.29 \pm 1.76 pins on the less affected side (effect of side, $F(1,19)=$ $10.075, P=.0050)$. Performance also improved from trial 1 to trial 2 (effect of trial, $F(1,19)=24.845, P<.0001$ ). In the bimanual task, we found improved performance during the week of experiments (ANOVA, effect of day, $F(4,76)=6.673$, $P=.0001)$ but no significant effect of trial.

One patient in the ON-group was not able to complete the pegboard task with the less affected hand or bimanually because of a ruptured flexor tendon in the thumb of the less affected hand.

Gait performance. There were no significant main effects or interactions concerning number of steps or total duration needed to complete the task.

\section{rTMS-Related Effects}

None of the rTMS protocols was able to induce marked changes in any measure after a single session. Only for the unimanual PPT did we find differential effects for either hand depending on the intervention protocol (Interaction Side $\times$ Intervention Protocol, $F(4,95)=2.802, P=.0301$; Interaction Side $\times$ Time $\times$ Intervention Protocol, $F(4,95)=2.802, P=.0301)$, with improved performance of the more affected side after any active rTMS protocol but not after sham (see Figure 3).

Statistical analysis did not show significant main effects or interactions with the intervention protocol for any other task, indicating that there was no difference of any active rTMS protocol compared with sham stimulation.

Analysis of baseline raw values showed only that the OFFgroup needed less steps on day 1 compared with the other days $($ Interaction Group $\times$ Day, $F(4,80)=2.547, P=.0456)$.

\section{Corticospinal Excitability}

As expected, RMT was higher than AMT (see Figure 4; ANOVA: effect of level of activation, $F(1,100)=401.772$, $P<.0001)$. We found higher RMT and lower AMT in the ON-group compared with the OFF-group (interaction of Level of Activation $\times$ Group, $F(1,100)=15.002, P=.0002)$ with
Figure 3

Boxplots for the Purdue Pegboard Task (PPT): Performance on the Unimanual Tasks for the Different Types of Intervention Separately for Either Group
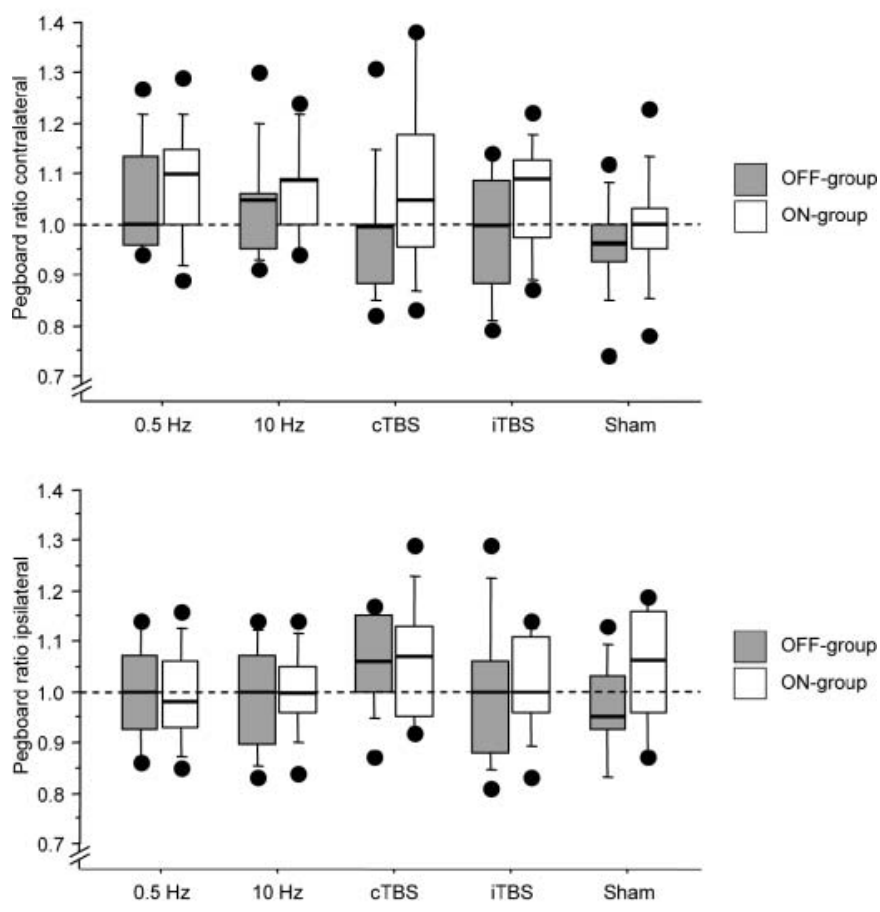

Note: The box represents all values within the 75th percentile with a horizontal bar at the position of the median, error bars at the 90th percentile, and single dots all values $>90$ th percentile. Values higher than 1 indicate improved performance after repetitive transcranial magnetic stimulation (rTMS) intervention. cTBS, continuous theta burst stimulation; iTBS, intermittent theta burst stimulation.

slightly decreased RMT and increased AMT after stimulation for both groups (Level of Activation $\times$ Time, $F(1,100)=$ $7.850, P=.0061)$. In the control ANOVA, for baseline values, we did not find any effect or interaction of day.

No correlation was found between motor thresholds, baseline EMG activity, and overall or limb rigidity.

\section{Mood Scale}

Mean values for the mood scale are summarized in Table 2. All patients reported decreased attention after the experiment (ANOVA, effect of time, $P=.0013$ ). The OFF-group reported more often that the stimulation had been effective than the ON-group (Chi-Square Test Efficacy $\times$ Group, $P=.045$ ). However, this was not related to the stimulation protocol. All other parameters did not show any significant effects.

Baseline values did not differ between groups or over the week of experiments. 


\section{Figure 4 \\ Effect of Group (OFF/ON) on RMT and AMT for the ADM of the More Affected Side in Percentage of Maximum Stimulator Output}

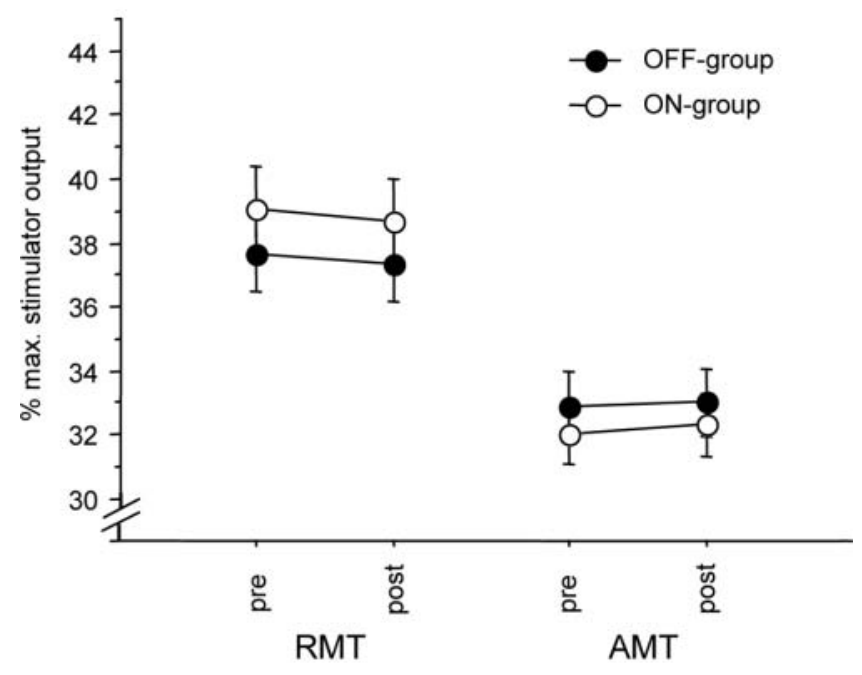

Note: Error bars indicate \pm 1 SEM. As there was no significant effect of type of intervention, data of all 5 sessions have been pooled. RMT, resting motor threshold; AMT, active motor threshold; ADM, abductor digiti minimi.

\section{UPDRS, BDI}

No significant main effects or interactions were found for UPDRS motor scores or BDI scores (see Table 3).

\section{Side Effects}

There were no serious side effects of TMS. Two patients reported transient headache following the experiments, and 1 patient reported nausea.

\section{Discussion}

The pivotal positive finding of this study is the marked and prolonged behavioral training effect of PD patients in the ON state, extending over the first 4 days of study. Dopamine is involved in motor planning, ${ }^{19}$ and motor learning can be enhanced by dopaminergic medication, particularly in the elderly. ${ }^{20}$ It is used for this purpose in some rehabilitative settings. ${ }^{21}$ Neurophysiological studies on direct-current stimulation and on paired associative stimulation have shown that dopaminergic input facilitates synapse-specific plasticity and reduces unspecific plasticity, thus focusing facilitation. ${ }^{22}$ However, the high degree of short-term (within 1 session) and long-term (from day to day) training effects in the arm motor tasks (pointing, pronation-supination, PPT) was unexpected as all tests were based on standard clinical tests. We are not aware of a study that has shown this extent and this duration of learning present over several days of training, even with rather conventional tasks.

We therefore did not include a group of patients who only received sham treatment for 5 consecutive days. It is hard to say whether the observed improvement of motor function reflects an unspecific rTMS effect or purely motor training. However, the marked and prolonged training effect is obviously dependent on dopaminergic input and has to be carefully considered for future clinical trials.

The pivotal negative finding of this study is that none of the rTMS protocols was strong enough to induce remarkable effects after a single session.

Against the background of several studies reporting beneficial effects of rTMS on motor performance in PD, some shortcomings in our study design have to be considered for the interpretation of these negative findings. The high degree of training and placebo effects might have masked subtle differential effects of the rTMS protocols used in this study. Moreover, there might have been interactions between the stimulation protocols as the experiments were conducted on consecutive days. We can therefore only comment on immediate short-term responses, whereas other authors stimulated patients with the same protocol in a sequence of several days or 4 weeks. ${ }^{23,24}$

In addition, it has been shown that PD patients are highly susceptible to chronic placebo effects ${ }^{25-27}$ and even show bilateral dopamine release in the dorsal and ventral striatum in response to placebo rTMS. ${ }^{28}$ However, a recent study on shortterm placebo effects in PD found an improvement in UPDRS scores after sham rTMS of about $21 \%$, which did not reach statistical significance, but there was a much more pronounced increase in subjective self-evaluation of motor function using a visual analog scale. ${ }^{29}$ Even mood changes might interfere with motor performance, and rTMS has been shown to be effective in the treatment of depression. ${ }^{30-32}$ Patients in the present study often reported after the experiment that they felt "somehow more at ease," but this could not be confirmed by the results of the mood scale and was attributed as a placebo effect. Interestingly, the OFF-group reported more often than the ON-group that they had found the stimulation effective, even if there was no change in motor performance. This might be explained by a "greater drive for symptom relief."28

Another important aspect is a potentially shared neuronal network responsible for motor learning as well as rTMS effects. On one hand, this might preclude distinct rTMS effects in patients who show impaired motor learning, as the OFF-group did in the present study. This is in line with a recent study showing that facilitating after effects of paired associative stimulation (PAS) could be found only in PD patients on medication but not in the same patients off medication. ${ }^{33}$ Thus, a dopaminergic pretreatment might be necessary for an effective rTMS. On the other hand, such a shared network could be a limit to neuroplastic changes, and intensive 
Table 2

Behavioral Self-Rating Scale, Mean Values

\begin{tabular}{lrrrr}
\hline & \multicolumn{2}{c}{ OFF } & \multicolumn{2}{c}{ ON } \\
\cline { 2 - 4 } & \multicolumn{1}{c}{ Pre } & Post & Pre & $0.49 \pm 1.78$ \\
Comfort & $-0.09 \pm 1.53$ & $0.22 \pm 1.27$ & $0.58 \pm 1.45$ & $0.64 \pm 1.65$ \\
Anxiety & $0.49 \pm 1.20$ & $0.53 \pm 1.09$ & $0.20 \pm 1.53$ & $0.76 \pm 1.31$ \\
Fatigue & $0.18 \pm 1.44$ & $-0.29 \pm 1.07$ & $0.62 \pm 1.23$ & $0.24 \pm 1.60$ \\
Mood & $0.44 \pm 1.00$ & $0.49 \pm 0.81$ & $0.89 \pm 1.26$ & $0.75 \pm 1.25$ \\
Irritation & $0.80 \pm 1.00$ & $0.80 \pm 1.00$ & $0.62 \pm 1.37$ \\
Attention & $0.24 \pm 1.32$ & $-0.13 \pm 1.14 *$ & $1.58 \pm 1.67$ & $-0.02 \pm 1.60^{*}$ \\
Pain & $1.04 \pm 1.89$ & $1.02 \pm 1.98$ & & $1.53 \pm 1.60$ \\
\hline
\end{tabular}

*Analysis of variance, effect of time, $P=.0013$.

Table 3

Mean UPDRS and BDI Values

\begin{tabular}{lccrr}
\hline & UPDRS Day 1 & UPDRS Day 5 & BDI Day 1 & BDI Day 5 \\
\hline OFF & $27.0 \pm 13.9$ & $30.3 \pm 11.1$ & $11.8 \pm 5.0$ & $11.5 \pm 5.4$ \\
ON & $26.7 \pm 10.4$ & $27.3 \pm 9.9$ & $9.8 \pm 7.6$ & $7.5 \pm 2.3$ \\
All & $26.7 \pm 12.0$ & $28.8 \pm 10.4$ & $10.8 \pm 6.4$ & $9.5 \pm 5.7$ \\
\hline
\end{tabular}

Abbreviations: BDI, Beck Depression Inventory; UPDRS, Unified Parkinson's Disease Rating Scale.

motor training might prevent the effects of rTMS applied directly after the training session. This has already been shown for PAS in healthy subjects. ${ }^{34,35}$ Thus, the intensive assessment of motor performance in the present study potentially constitutes a confounding factor, especially for the ON-group, which showed clear training effects.

Recently, it has been shown that repeated sessions of rTMS at a frequency of $25 \mathrm{~Hz}$ are capable of inducing stronger and longer lasting effects on motor performance than singlesession rTMS. ${ }^{23,24}$ In addition, in these studies, several motor areas were stimulated in each session. This presumably indicates that more intensive rTMS protocols, together with sufficient times for consolidation of the effects, are crucial to overcome mechanisms of maladaptive plasticity or simply an impaired susceptibility to the external induction of neuroplastic changes to reach a therapeutic value.

\section{Conclusions}

This study shows a high degree of training effects even in standard clinical assessment methods that outweigh possible effects of a single session of rTMS over the hand motor area. This emphasizes the importance of a negative control condition for TMS (sham stimulation) as well as careful and prolonged pretraining of motor tasks to avoid interference with rTMS interventions. The high hopes that were connected with the introduction of the TBS protocol could not be confirmed for short-term effects following a single session. For future studies with a therapeutic intention, a design of repeated rTMS sessions seems most promising.

\section{References}

1. Siebner HR, Peller M, Willoch F, et al. Lasting cortical activation after repetitive TMS of the motor cortex: a glucose metabolic study. Neurology. 2000;54:956-963.

2. Siebner HR, Rothwell J. Transcranial magnetic stimulation: new insights into representational cortical plasticity. Exp Brain Res. 2003;148:1-16.

3. Strafella AP, Paus T, Fraraccio M, Dagher A. Striatal dopamine release induced by repetitive transcranial magnetic stimulation of the human motor cortex. Brain. 2003;126(pt 12):2609-2615.

4. Sommer M, Kamm T, Tergau F, Ulm G, Paulus W. Repetitive pairedpulse transcranial magnetic stimulation affects corticospinal excitability and finger tapping in Parkinson's disease. Clin Neurophysiol. 2002;113: 944-950.

5. Lefaucheur JP, Drouot X, Von Raison F, Menard-Lefaucheur I, Cesaro P, Nguyen JP. Improvement of motor performance and modulation of cortical excitability by repetitive transcranial magnetic stimulation of the motor cortex in Parkinson's disease. Clin Neurophysiol. 2004;115:2530-2541.

6. Pascual-Leone A, Valls-Sole J, Brasil-Neto JP, Cammarota A, Grafman J, Hallett M. Akinesia in Parkinson's disease: II. Effects of subthreshold repetitive transcranial motor cortex stimulation. Neurology. 1994;44:892-898.

7. Tergau F, Wassermann EM, Paulus W, Ziemann U. Lack of clinical improvement in patients with Parkinson's disease after low and high frequency repetitive transcranial magnetic stimulation. Electroencephalogr Clin Neurophysiol Suppl. 1999;51:281-288.

8. Fregni F, Simon DK, Wu A, Pascual-Leone A. Non-invasive brain stimulation for Parkinson's disease: a systematic review and meta-analysis of the literature. J Neurol Neurosurg Psychiatry. 2005;76:1614-1623.

9. Helmich RC, Siebner HR, Bakker M, Munchau A, Bloem BR. Repetitive transcranial magnetic stimulation to improve mood and motor function in Parkinson's disease. J Neurol Sci. 2006;248(1-2):84-96.

10. Sommer M, Paulus W. Pulse configuration and rTMS efficacy: a review of clinical studies. Suppl Clin Neurophysiol. 2003;56:33-41.

11. Huang YZ, Edwards MJ, Rounis E, Bhatia KP, Rothwell JC. Theta burst stimulation of the human motor cortex. Neuron. 2005;45:201-206.

12. Paulus W. Toward establishing a therapeutic window for rTMS by theta burst stimulation. Neuron. 2005;45:181-183.

13. Jancke L, Steinmetz H, Benilow S, Ziemann U. Slowing fastest finger movements of the dominant hand with low-frequency rTMS of the hand area of the primary motor cortex. Exp Brain Res. 2004;155:196-203.

14. Beck AT, Ward CH, Mendelson M, Mock J, Erbaugh J. An inventory for measuring depression. Arch Gen Psychiatry. 1961;4:561-571.

15. Defer GL, Widner H, Marie RM, Remy P, Levivier M. Core assessment program for surgical interventional therapies in Parkinson's disease (CAPSIT-PD). Mov Disord. 1999;14:572-584.

16. Lisanby SH, Gutman D, Luber B, Schroeder C, Sackeim HA. Sham TMS: intracerebral measurement of the induced electrical field and the induction of motor-evoked potentials. Biol Psychiatry. 2001;49:460-463. 
17. Fahn S, Elton R, Committee MotUD. Unified Parkinson's Disease Rating Scale. In: Fahn S, Marsden CD, Calne DB, Goldstein M, eds. Recent Developments in Parkinson's Disease. Vol. 2. Florham Park, NJ: Macmillan Health Care Information; 1987:153-304.

18. Rothwell JC, Hallett M, Berardelli A, Eisen A, Rossini P, Paulus W. Magnetic stimulation: motor evoked potentials. The International Federation of Clinical Neurophysiology. Electroencephalogr Clin Neurophysiol Suppl. 1999;52:97-103.

19. Garraux G, Peigneux P, Carson RE, Hallett M. Task-related interaction between basal ganglia and cortical dopamine release. J Neurosci. 2007; 27:14434-14441.

20. Floel A, Vomhof P, Lorenzen A, Roesser N, Breitenstein C, Knecht S. Levodopa improves skilled hand functions in the elderly. Eur J Neurosci. 2008;27:1301-1307.

21. Scheidtmann K, Fries W, Muller F, Koenig E. Effect of levodopa in combination with physiotherapy on functional motor recovery after stroke: a prospective, randomised, double-blind study. Lancet. 2001;358:787-790.

22. Kuo MF, Paulus W, Nitsche MA. Boosting focally-induced brain plasticity by dopamine. Cereb Cortex. 2008;18:648-651.

23. Lomarev MP, Kanchana S, Bara-Jimenez W, Iyer M, Wassermann EM, Hallett M. Placebo-controlled study of rTMS for the treatment of Parkinson's disease. Mov Disord. 2006;21:325-331.

24. Khedr EM, Rothwell JC, Shawky OA, Ahmed MA, Hamdy A. Effect of daily repetitive transcranial magnetic stimulation on motor performance in Parkinson's disease. Mov Disord. 2006;21:1311-1316.

25. Goetz CG, Leurgans S, Raman R, Stebbins GT. Objective changes in motor function during placebo treatment in PD. Neurology. 2000;54:710-714.

26. McRae C, Cherin E, Yamazaki TG, et al. Effects of perceived treatment on quality of life and medical outcomes in a double-blind placebo surgery trial. Arch Gen Psychiatry. 2004;61:412-420.
27. de la Fuente-Fernandez R, Ruth TJ, Sossi V, Schulzer M, Calne DB, Stoessl AJ. Expectation and dopamine release: mechanism of the placebo effect in Parkinson's disease. Science. 2001;293:1164-1166.

28. Strafella AP, Ko JH, Monchi O. Therapeutic application of transcranial magnetic stimulation in Parkinson's disease: the contribution of expectation. Neuroimage. 2006;31:1666-1672.

29. Fregni F, Boggio PS, Bermpohl F, et al. Immediate placebo effect in Parkinson's disease: is the subjective relief accompanied by objective improvement? Eur Neurol. 2006;56:222-229.

30. Chistyakov AV, Kaplan B, Rubichek O, et al. Antidepressant effects of different schedules of repetitive transcranial magnetic stimulation vs. clomipramine in patients with major depression: relationship to changes in cortical excitability. Int J Neuropsychopharmacol. 2005; 8(2):223-233.

31. George MS, Nahas Z, Molloy M, et al. A controlled trial of daily left prefrontal cortex TMS for treating depression. Biol Psychiatry. 2000; 48(10):962-970.

32. Fregni F, Santos CM, Myczkowski ML, et al. Repetitive transcranial magnetic stimulation is as effective as fluoxetine in the treatment of depression in patients with Parkinson's disease. J Neurol Neurosurg Psychiatry. 2004;75:1171-1174.

33. Ueki Y, Mima T, Kotb MA, et al. Altered plasticity of the human motor cortex in Parkinson's disease. Ann Neurol. 2006;59:60-71.

34. Stefan K, Wycislo M, Gentner R, et al. Temporary occlusion of associative motor cortical plasticity by prior dynamic motor training. Cereb Cortex. 2006;16:376-385.

35. Ziemann U, Ilic TV, Pauli C, Meintzschel F, Ruge D. Learning modifies subsequent induction of long-term potentiation-like and long-term depression-like plasticity in human motor cortex. J Neurosci. 2004;24: $1666-1672$. 